\title{
Evaluation of 2 electronic handheld devices for diagnosis of ketonemia and glycemia in dairy goats
}

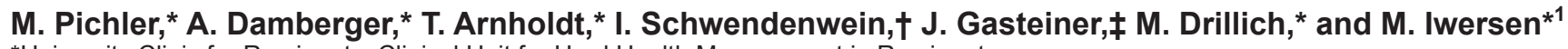 \\ *University Clinic for Ruminants, Clinical Unit for Herd Health Management in Ruminants, \\ Department for Farm Animals and Veterinary Public Health, and \\ †Central Clinical Pathology Unit, Department for Pathobiology, University of Veterinary Medicine Vienna, 1210 Vienna, Austria \\ $\ddagger$ Agricultural Research and Education Center Raumberg-Gumpenstein, 8952 Irdning, Styria, Austria
}

\begin{abstract}
The objective of this study was to evaluate the suitability of the electronic handheld devices FreeStyle Precision (FSP; Abbott Germany, Wiesbaden, Germany) and GlucoMen LX Plus (GML; A. Menarini GmbH, Vienna, Austria) for the measurement of $\beta$-hydroxybutyrate (BHBA) in whole blood in dairy goats. Additionally, glucose concentration was analyzed with the FSP device. For method comparison, the samples were also analyzed in the laboratory by standard methods, which served as the gold standard in our study. A further objective was to evaluate the agreement between samples obtained by minimal invasive venipuncture of an ear vein and measurements of whole blood samples from the jugular vein (vena jugularis). In total, 173 blood sample pairs collected from 28 goats were obtained from an ear vein and from the jugular vein. The Spearman correlation coefficients $\left(\mathrm{r}_{\mathrm{sp}}\right)$ for BHBA concentrations determined with the FSP or GML and the gold standard were 0.95 and 0.85 for the ear vein and 0.98 and 0.88 for the jugular vein, respectively. Bland-Altman plots of differences showed a positive bias of 0.12 (ear vein) and 0.21 (jugular vein) when determination was performed with the FSP and a negative bias of 0.21 (ear vein) and 0.24 (jugular vein) when using the GML. For the FSP, applying the adjusted thresholds determined by ROC analysis of 0.9 (ear vein) and $1.0 \mathrm{mmol} / \mathrm{L}$ (jugular vein) allowed to distinguish between healthy goats and animals with hyperketonemia with sensitivities (Se) and specificities (Sp) for samples from the ear vein of 0.98 and 0.85 , and from the jugular vein of 0.99 and 0.94 , respectively. For the GML, adjusted thresholds were $0.5 \mathrm{mmol} / \mathrm{L}$ for the ear vein $(\mathrm{Se}=0.94, \mathrm{Sp}=0.75)$ and $0.6 \mathrm{mmol} / \mathrm{L}$ for the jugular vein $(\mathrm{Se}=0.88, \mathrm{Sp}=0.91)$. Repeated analyses of defined whole blood samples showed average inter- and intraassay coefficients of variation of 6.6
\end{abstract}

Received April 2, 2014.

Accepted August 15, 2014.

${ }^{1}$ Corresponding author: Michael.Iwersen@vetmeduni.ac.at and $7.3 \%$ for FSP, and of 35.6 and $35.4 \%$ for GML, respectively. Test characteristics for determining glucose concentration with the FSP compared with the gold standard were poor (ear vein: $\mathrm{r}_{\mathrm{sp}}=0.41$; jugular vein: $\mathrm{r}_{\mathrm{sp}}=0.51$ ), with low validity to distinguish between hypo- and normoglycemia ( $\mathrm{Se}=0.71, \mathrm{Sp}=0.66)$. The present study showed good test characteristics for the FSP and moderate for the GML device for determining BHBA concentrations in whole blood. Additionally the results demonstrate the suitability of testing BHBA concentration in a blood drop obtained from an ear vein. Based on the results of this study, the FSP device is not suitable to differentiate normo- from hypoglycemia in goats.

Key words: goat, pregnancy toxemia, ketosis, handheld meter

\section{INTRODUCTION}

Disorders in the carbohydrate and fat metabolism are very common and can affect most species. In dairy goats, clinical ketosis manifests predominantly in early lactation (Stelletta et al., 2008). Does carrying multiple fetuses, undernourished dams, or animals in a very good body condition are predisposed to clinical ketosis (Rook, 2000; Brozos et al., 2011). Clinical cases of ketosis usually occur sporadically and unpredictably and can affect up to $2 \%$ of the herd (Rook, 2000). Mortality rates of the dams can reach up to $80 \%$ in the case of a problem within a herd (Andrews, 1997; Rook, 2000). Early detection of subclinical ketosis followed by an efficacious treatment should help to minimize economic losses (Rook, 2000; Sargison, 2007; Brozos et al., 2011).

An increased concentration of BHBA and low or physiological blood glucose concentrations are diagnostic hallmarks for ketosis (Andrews, 1997; Rook, 2000). To our knowledge, no validated thresholds to define hyperketonemia (HKET) in goats, showing negative effects on animal health or animal performance, have been reported yet. Hence, thresholds based on clinical experience in ewes as reported in the literature were used as decision criterion for HKET in the current study. 
Physiological BHBA concentrations in ewes are defined as serum concentrations $<0.8 \mathrm{mmol} / \mathrm{L}$ (Rook, 2000). Concentrations of BHBA $\geq 0.8 \mathrm{mmol} / \mathrm{L}$ are indicative for maladaptation of the energy metabolism leading to impaired energy balance (Navarre et al., 2012). Animals with serum BHBA concentrations between 0.8 to $1.6 \mathrm{mmol} / \mathrm{L}$ are in a negative energy balance and thus at greater risk to develop clinical ketosis (Navarre et al., 2012). Animals with BHBA concentrations $>1.6 \mathrm{mmol} / \mathrm{L}$ are in severe negative energy balance and, in combination with clinical symptoms, suffering from clinical ketosis (Rook, 2000). A study by Ismail et al. (2008) showed that pregnant does with subclinical ketosis had significantly lower glucose concentrations compared with nonketotic does. Whereas, in dairy cows, dipsticks can be used for detecting ketone bodies excreted in urine or milk (Carrier et al., 2004; Krogh et al., 2011), in small ruminants these semiquantitative tests are not as accurate as blood tests (Brozos et al., 2011). Electronic hand-held devices showed excellent test characteristics for measurement of BHBA and to some extent for glucose in whole blood in humans (Byrne et al., 2000; Noyes et al., 2007), cows (Voyvoda and Erdogan, 2010; Iwersen et al., 2013), and sheep (Panousis et al., 2012; Pichler et al., 2014). Recently, Doré et al. (2013) found an excellent correlation $(\mathrm{r}=$ 0.98; $P<0.01$ ) between BHBA concentrations determined in whole blood of goats with the handheld meter Precision Xtra (Abbott Diabetes Care, Saint-Laurent, Canada) and serum BHBA concentrations analyzed at a laboratory.

The objective of the present study was to evaluate 2 electronic handheld devices, FreeStyle Precision (FSP; Abbott Germany, Wiesbaden, Germany) and GlucoMen LX Plus (GML; A. Menarini GmbH, Vienna, Austria), for on-site diagnosis of hyperketonemia in dairy goats. Additionally, the FSP was tested for measuring glucose concentrations in blood. A further objective was to evaluate the agreement between the results of BHBA and glucose concentrations in a single blood drop obtained from an ear vein and concentrations in whole blood from the jugular vein.

\section{MATERIALS AND METHODS}

\section{Animals}

The study was conducted on 28 White German milk goats (a composite of local white breeds and Saanen) commonly kept in Austria and Germany. The animals were owned by the Agricultural Research and Education Center Raumberg-Gumpenstein (Styria, Austria) and housed in 2 groups of 13 and 15 animals on deep litter. The goats had access to pasture during spring and summer, and were fed twice per day with $0.6 \mathrm{~kg}$ of hay and $0.6 \mathrm{~kg}$ of concentrates per meal (18\% soybean meal, $82 \%$ rolled barley grain). Before parturition, the does were kept separated in single boxes but with contact to the herd. After kidding, the does were regrouped. The goats were milked twice daily in a sideby-side milking parlor. The average milk yield of the herd was $924 \mathrm{~kg}$ per lactation with an average milk fat and protein content of 2.62 and $2.77 \%$, respectively. All does within 5 wk before and up to 9 wk after parturition were enrolled in the study and checked visually for any health disorders before sampling. The study was approved by the Austrian Federal State Styria (FA10A78Gu-15/2011-2) according to the Austrian Law for Animal Experiments.

\section{Sampling Procedures and Measurement of BHBA and Glucose Concentrations}

The present study was part of a longitudinal survey in dairy goats, where sampling was performed once a week starting 5 wk before to $6 \mathrm{wk}$ after kidding, followed by an additional blood sample on wk 9 postpartum. Only a subset of these samples, collected in the kidding season between January to May 2012, were used to evaluate the handheld devices. Hence, individual animals were tested between 4 to 8 times within the study period. The metabolite BHBA was determined with the handheld devices FSP and GML in blood obtained by minimal invasive venipuncture of an ear vein and in whole blood from the jugular vein. Additionally, the glucose concentration was determined with the FSP at both sampling sites. In contrast to the already evaluated Precision Xtra device (Doré et al., 2013), the FSP and GML operate without prior calibration procedures.

For sampling procedures, the animal and animal's head were fixed by one person while a second person performed the venipuncture of an ear vein. For this, a well-visible vein was chosen and punctured with a cannula (Erosa Injektionskanüle, $1.2 \times 50 \mathrm{~mm}$, Rose $\mathrm{GmbH}$, Trier, Germany), as described previously in ewes by Pichler et al. (2014). Performing this technique, a drop of whole blood of at least $0.60 \mu \mathrm{L}$ was obtained and immediately analyzed with both handheld meters. Measurement procedures were repeated immediately in a blood sample obtained from the jugular vein, using a blood-collection tube system (Vacuette, Greiner Bio-One GmbH, Kremsmünster, Austria), serum tubes (Vacuette, Z-Serum Clot Activator, $9 \mathrm{~mL}$, Greiner BioOne $\mathrm{GmbH}$ ), and a 20-gauge needle (Vacuette $0.9 \times 38$ $\mathrm{mm}$, Greiner Bio-One GmbH). Measurement of BHBA was performed with the FSP and GML using FreeStyle Precision $\beta$-ketone strips (Abbott Diabetes Care, Witney, UK) and GlucoMen LX $\beta$-ketone sensor strips (A. 
Menarini $\mathrm{GmbH}$ ), respectively. Glucose was measured only with the FSP using the FreeStyle Precision glucose strips (Abbott Diabetes Care, Witney, UK) because the glucose test strips for the GML were not commercially available during most of the time of the study period. After blood sampling from the jugular vein, the sensor of the test strips were dipped directly onto the surface of the blood in the tubes for determining the BHBA and glucose concentration. After blood application on the test strips, BHBA concentrations were presented at the display of the FSP and GML after $10 \mathrm{~s}$; glucose concentration was displayed at the FSP after $5 \mathrm{~s}$.

For method comparison, the samples obtained from the jugular vein were also analyzed on a conventional large-scale analyzer for clinical chemistry at the laboratory of the Central Clinical Pathology Unit (CCPU), University of Veterinary Medicine Vienna (Vetmeduni Vienna, Austria). After approximately $2 \mathrm{~h}$ of clotting at ambient temperature (between 4 and $10^{\circ} \mathrm{C}$ ), samples were centrifuged at $2,200 \times g$ for $10 \mathrm{~min}$ at room temperature (Eppendorf Centrifuge 5804, Eppendorf AG, Hamburg, Germany) and serum was separated and stored in 2 aliquots $(2 \mathrm{~mL}$, Micro tube, Sarstedt, Nümbrecht, Germany) at $-25^{\circ} \mathrm{C}$ until analysis at the CCPU. Serum was analyzed with an automated wet chemistry analyzer Cobas 6000/c501 (Roche Diagnostics GmbH, Vienna, Austria). The BHBA concentration was determined by a colorimetric enzymatic test (Ranbut, D-3-hydroxybutyrate; Randox Laboratories Ltd., Crumlin, UK). This method is based on the oxidation of D-3-hydroxybutyrate to acetoacetate by the enzyme 3-hydroxybutyrate-dehydrogenase. The oxidation is coupled to an NAD indicator system. The induced color change is proportional to the BHBA concentration.

The serum glucose concentration was determined by an enzymatic colorimetric hexokinase method. Hexokinase catalyzes the phosphorylation of glucose to glucose-6-phosphate and adenosine-diphosphate by ATP. This reaction is coupled to an NADP color indicator system. The concentrations of BHBA and glucose determined in serum at the CCPU were defined as the gold standard in our study. The coefficients of variation were calculated for the wet chemistry methods used at the laboratory.

\section{Evaluation of Precision}

An additional aim of the current study was to evaluate the precision of BHBA measurements in whole blood with the 2 electronic handheld meters. For this, 3 blood samples with different BHBA concentrations (based on FSP measurements) of 0.4 (representing a physiological concentration: $<0.8 \mathrm{mmol} / \mathrm{L}$ ), 1.3 (representing moderate HKET: 0.8 to $1.6 \mathrm{mmol} / \mathrm{L}$ ), and
$1.6 \mathrm{mmol} / \mathrm{L}$ (representing severe HKET: $>1.6 \mathrm{mmol} / \mathrm{L}$ ) were analyzed with 10 different FSP and GML meters to calculate their specific interassay CV. To calculate the intraassay $\mathrm{CV}$, each of the 3 blood samples was analyzed 10 times using a single FSP and GML device.

\section{Statistical Analysis}

Data were analyzed using the software packages PASW Statistics (version 17, IBM, Ehningen, Germany), MedCalc (version 12.7.7, MedCalc Software, Ostend, Belgium), and BiAS (version 9.07, EpsilonVerlag, Darmstadt, Germany). The level of significance for all statistical tests was set at $P<0.05$. The associations between the gold standard concentrations (serum BHBA and glucose) and the concentrations determined with the handheld devices in the blood drop of an ear vein and in whole blood from the jugular vein were calculated as Spearman correlation coefficients.

The agreement of the results between the handheld meters and the laboratory results were evaluated by Bland-Altman plots (Bland and Altman, 1986). The corresponding means, standard deviations, and binomial $95 \%$ confidence interval $\left(\mathbf{C I}_{\mathbf{9 5}}\right)$ were calculated.

Based on serum BHBA concentrations determined at the CCPU, samples were classified as physiologic $(<0.8 \mathrm{mmol} / \mathrm{L})$, moderate HKET $(0.8$ to $1.6 \mathrm{mmol} / \mathrm{L})$, and severe HKET $(>1.6 \mathrm{mmol} / \mathrm{L})$. According to the results of glucose determination at the CCPU, samples were classified as hypoglycemic $(\leq 49 \mathrm{mg} / \mathrm{dL})$, normoglycemic (50 to $63 \mathrm{mg} / \mathrm{dL}$ ), and hyperglycemic (>63 mg/dL; Schwendenwein, 2009). Based on these classifications and thresholds, sensitivity (Se), specificity $(\mathbf{S p})$, and the Youden index $(\mathbf{J})$ were calculated. The $\mathrm{J}$, reflecting the number of all correctly identified outcomes, is calculated as $\mathrm{Se}+\mathrm{Sp}-1$ (Youden, 1950).

Receiver operating characteristic (ROC) analyses were performed for adjusting the thresholds for the handheld devices (based on the laboratory results) to identify moderate and severe HKET, as well as to identify hypo- and hyperglycemia. The resulting area under the ROC curve (AUC) demonstrates the quality of the thresholds based on Se and Sp (Swets, 1988). Inter- and intraassay CV were determined to analyze the precision of the measurements of the 2 handheld meters. The CV was calculated as SD divided by the mean times 100. A one-way ANOVA (with Bonferroni post-hoc test) was assessed to evaluate differences between the glucose concentrations within the 3 BHBA classes.

\section{RESULTS}

A total of 173 pairs of blood samples (ear vein, jugular vein) were collected from 28 goats, with 154 
sample pairs (89\%) obtained from lactating does and $19(11 \%)$ from nonlactating does in the last trimester of gestation. None of the animals showed any clinical sign of ketosis. All samples were analyzed for the concentration of BHBA with the FSP, GML, and at the CCPU. In 1 sample, the glucose concentration analyzed at the CCPU was below the minimum analytical detection limit. This sample was excluded from statistical analyses; hence, 172 pairs of samples were included in the analyses regarding glucose concentrations. The $\mathrm{CV}$ of the wet chemistry methods used at the CCPU were $3.6 \%$ for BHBA and $1.9 \%$ for glucose measurements.

\section{Concentration of BHBA}

The median BHBA concentration of all samples determined at the CCPU was $0.82 \mathrm{mmol} / \mathrm{L}$, with an interquartile range of $0.51 \mathrm{mmol} / \mathrm{L}$. Based on the laboratory results, $49 \%(\mathrm{n}=85)$ of the samples were classified as physiological, $45 \%(\mathrm{n}=77)$ as moderate hyperketonemic, and 6\% (n = 11) as severe hyperketonemic. All samples with a serum BHBA concentration $>0.8 \mathrm{mmol} / \mathrm{L}$ were obtained from lactating does. The BHBA and glucose concentrations, stratified by sampling time, are presented as boxplots in Figure 1. Samples collected antepartum up to wk 5 postpartum showed contrary alterations in BHBA and glucose concentrations. Approximately 27\% (24 of 88) of the samples classified as HKET (BHBA $\geq 0.8 \mathrm{mmol} / \mathrm{L}$ ) were additionally classified as hypoglycemic (glucose $\leq 49 \mathrm{mg} / \mathrm{dL}$ ).

The Spearman correlation coefficient $\left(\mathbf{r}_{\mathrm{sp}}\right)$ for the laboratory BHBA results and the concentration measured with the FSP was 0.95 (ear vein) and 0.98 (jugular vein), respectively (both $P<0.01$ ). The $\mathrm{r}_{\mathrm{sp}}$ for the laboratory BHBA concentration and the results determined with the GML were 0.85 (ear vein) and 0.88 (jugular vein), respectively (both $P<0.01$ ). The $\mathrm{r}_{\mathrm{sp}}$ for the BHBA concentration from whole blood of an ear vein and from the jugular vein determined with the FSP and GML were 0.94 and 0.85 , respectively (both $P<0.01)$.

Figures 2 and 3 show Bland-Altman plots for differences between the gold standard (CCPU) and BHBA values determined with the FSP and GML. For the FSP, mean differences between CCPU and samples obtained from an ear vein and from the jugular vein were $-0.12\left(\mathrm{CI}_{95}=-0.48\right.$ to 0.23$)$ and $-0.21 \mathrm{mmol} / \mathrm{L}$ $\left(\mathrm{CI}_{95}=-0.59\right.$ to 0.18). For the GML, mean differences between the gold standard and samples from an ear vein and from the jugular vein were $0.21\left(\mathrm{CI}_{95}=-0.26\right.$ to 0.68$)$ and $0.24 \mathrm{mmol} / \mathrm{L}\left(\mathrm{CI}_{95}=-0.10\right.$ to 0.58$)$.

Based on the previous defined thresholds for moderate and severe HKET, ROC analyses were performed to
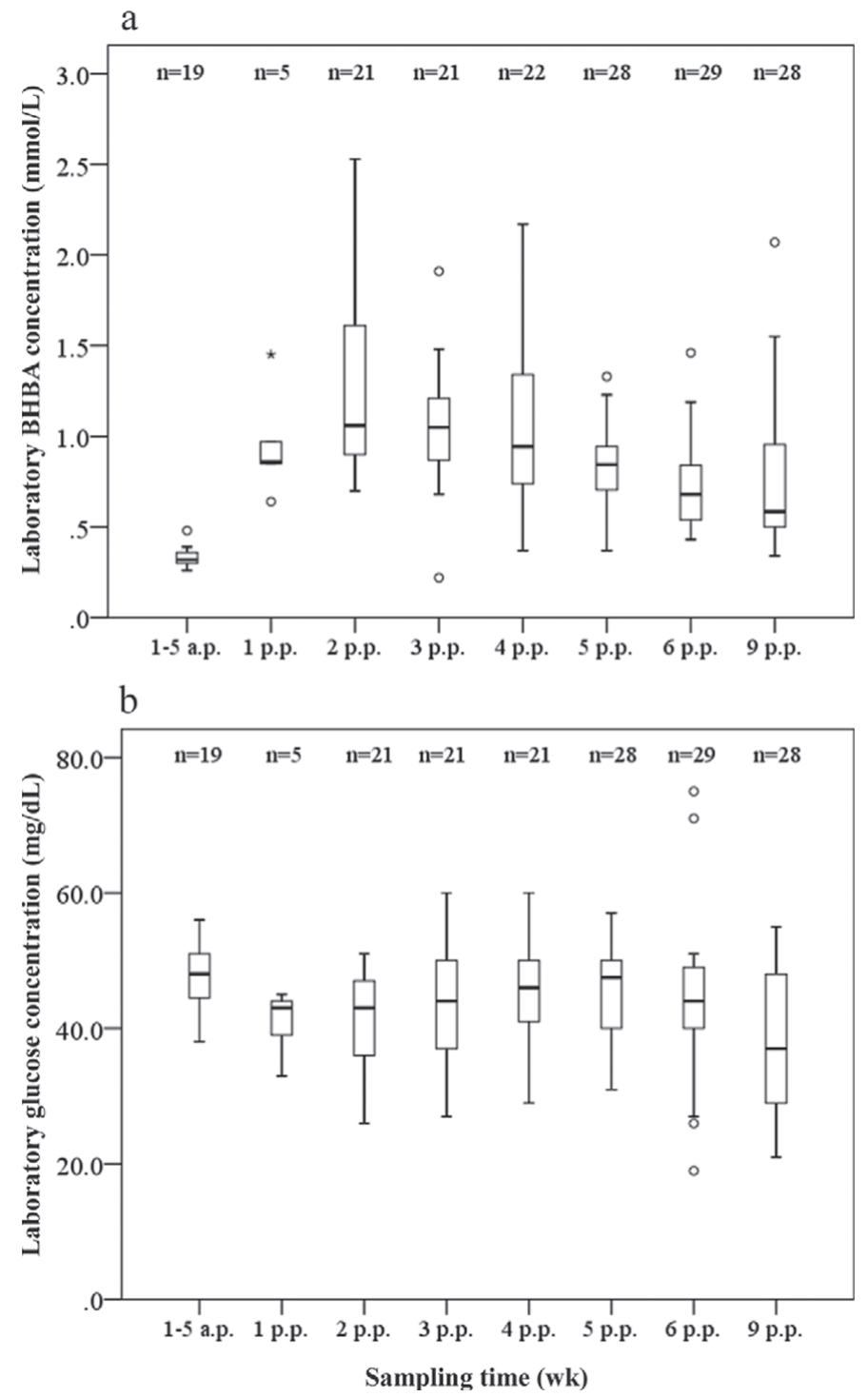

Figure 1. Box plots presenting the BHBA $(\mathrm{a} ; \mathrm{n}=173)$ and glucose (b; $\mathrm{n}=172$ ) concentrations determined at the laboratory antepartum (a.p.) and postpartum (p.p.). The heavy black line inside each box marks the median (50th percentile); lower and upper hinges mark the 25th and 75th percentiles, respectively. Whiskers end at the smallest and largest statistical values that are not outliers; outliers and extreme values are designated by an open circle $(\bigcirc)$.

identify best thresholds for both handheld meters. The adjusted thresholds for the FSP and GML are presented in Table 1. To distinguish between healthy animals (BHBA $<0.8 \mathrm{mmol} / \mathrm{L}$ ) and does with moderate HKET (BHBA between 0.8 and $1.6 \mathrm{mmol} / \mathrm{L}$ ), the optimized cutoff value for samples obtained from an ear vein with the FSP was $0.9 \mathrm{mmol} / \mathrm{L}(\mathrm{Se}=0.98 ; \mathrm{Sp}=0.85)$. For using the GML to distinguish between physiological and moderately high BHBA concentrations in blood from an ear vein, an optimized threshold of $0.5 \mathrm{mmol} / \mathrm{L}$ was calculated $(\mathrm{Se}=0.94 ; \mathrm{Sp}=0.75)$. Table 1 presents 

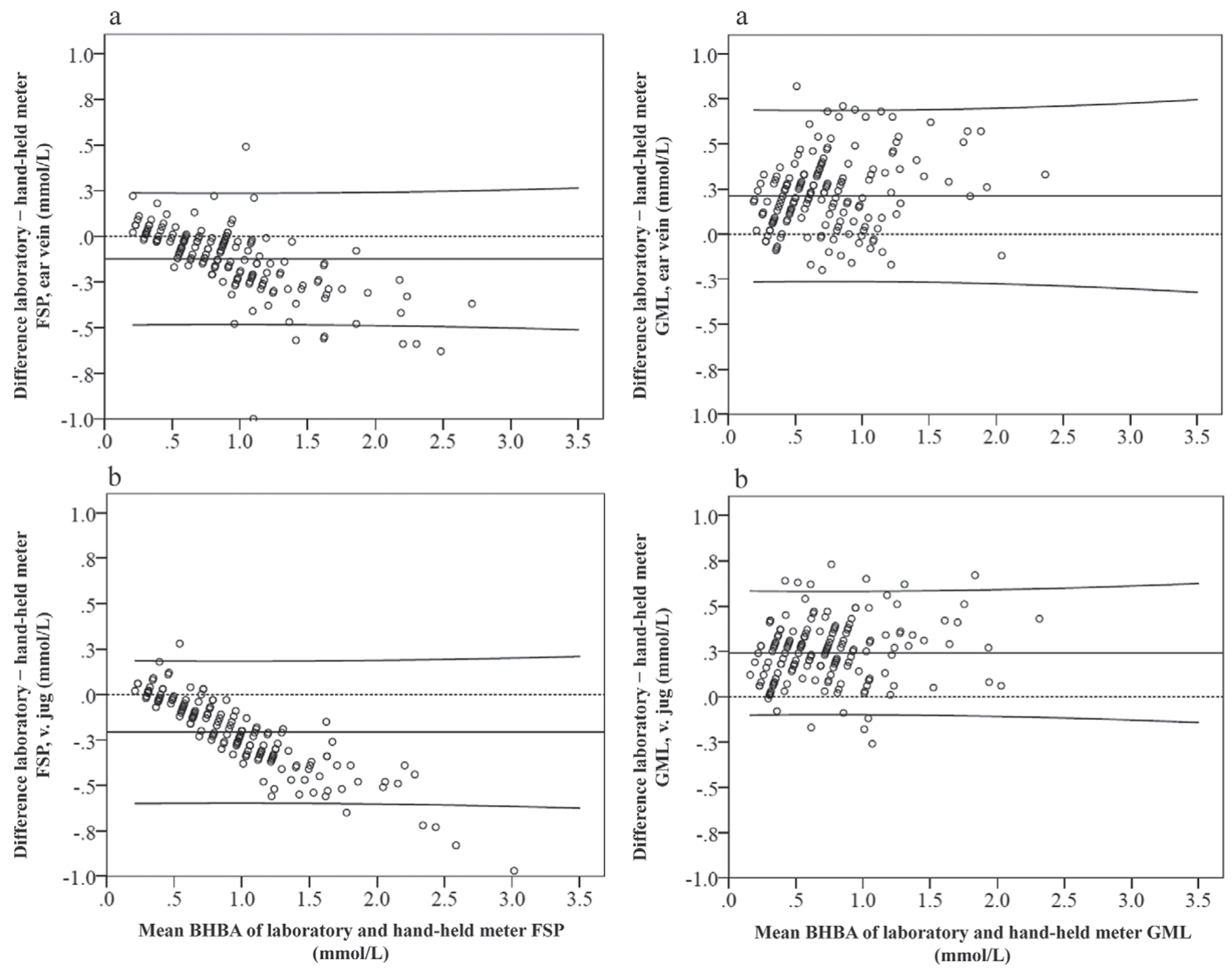

Figure 2. Bland-Altman plot of differences for the FreeStyle Precision handheld device (FSP; Abbott Diabetes Care Inc., Abbott Park, IL) to evaluate the BHBA concentration of the whole blood from an ear vein (a) and from the vena jugularis (v. jug.; b). The solid line in the middle shows the mean; the upper and lower solid lines present the mean $\pm \mathrm{SD}$.

also the results for the jugular vein and additional test characteristics (AUC, J).

\section{Concentration of Glucose}

The median of the glucose concentration determined at the CCPU was $45.0 \mathrm{mg} / \mathrm{dL}$, with an interquartile range of $11.8 \mathrm{mg} / \mathrm{dL}$. Overall, $75.0 \%(\mathrm{n}=129)$ of the samples showed serum glucose concentrations $\leq 49 \mathrm{mg} / \mathrm{dL}$ (hypoglycemic), whereas $23.8 \%$ ( $\mathrm{n}=41$ ) were within the physiological range $(50$ to $63 \mathrm{mg} / \mathrm{dL}$ ) and 2 samples $(1.2 \%)$ were found with concentrations

Figure 3. Bland-Altman plot of differences for the GlucoMen LX Plus handheld device (GML; A. Menarini GmbH, Vienna, Austria) to evaluate the BHBA concentration of the whole blood from an ear vein (a) and from the vena jugularis (v. jug.; b). The solid line in the middle shows the mean; the upper and lower solid lines present the mean $\pm \mathrm{SD}$.

$>63 \mathrm{mg} / \mathrm{dL}$ (hyperglycemic). Glucose concentrations in samples classified as normoketotic $(<0.8 \mathrm{mmol} / \mathrm{L})$ were $3.87 \mathrm{mg} / \mathrm{dL}$ greater than in samples classified as moderate HKET $(P=0.02)$ and $8.87 \mathrm{mg} / \mathrm{dL}$ greater compared with samples classified as severe HKET $(P$ $<0.01)$.

The Spearman correlation coefficient for glucose concentrations measured at the CCPU and in a blood drop from an ear vein with the FSP was $0.41(P<0.01)$; the correlation coefficient for the gold standard and whole blood from the jugular vein was $\mathrm{r}_{\mathrm{sp}}=0.51(P<0.01)$. The correlation coefficient for glucose concentrations 
Table 1. Receiver operating characteristic (ROC) analysis for the handheld meters Freestyle Precision (FSP; Abbott Diabetes Care Inc., Abbott Park, IL) and GlucoMen LX Plus (GML; A. Menarini GmbH, Vienna, Austria) of whole blood from an ear vein and from the vena jugularis in dairy goats (samples, $\mathrm{n}=173$ )

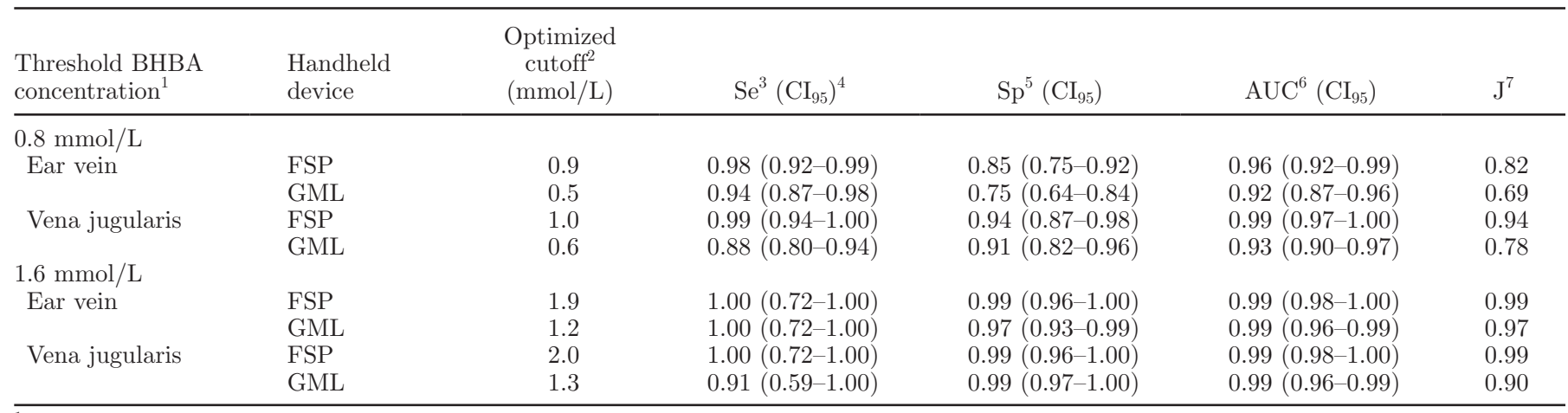

${ }^{1}$ Serum BHBA concentration determined at the laboratory.

${ }^{2}$ Optimized cutoff value for the FreeStyle Precision and GlucoMen LX Plus devices.

${ }^{3}$ Sensitivity $=$ percentage of ketotic does that tested positive.

${ }^{4} \mathrm{CI}_{95}=95 \%$ confidence interval.

${ }^{5}$ Specificity $=$ percentage of nonketotic does that tested negative.

${ }^{6} \mathrm{AUC}=$ area under the ROC curve.

${ }^{7}$ Youden index $=(\mathrm{Se}+\mathrm{Sp}-1)$; deviations are caused by rounding.

determined with the FSP from whole blood of an ear vein and the jugular vein was $\mathrm{r}_{\mathrm{sp}}=0.74(P<0.01)$. The Bland-Altman plots in Figure 4 illustrate the mean differences between the laboratory results and the FSP in samples obtained from an ear vein $\left(-6.3 \mathrm{mg} / \mathrm{dL} ; \mathrm{CI}_{95}\right.$ $=-26.5$ to 13.9$)$ and from the jugular vein $(-10.9 \mathrm{mg} /$ $\mathrm{dL} ; \mathrm{CI}_{95}=-28.1$ to 6.2 ).

To calculate optimized thresholds for determining glucose concentration with the FSP, ROC analyses were performed. For identifying hypo- and hyperglycemic goats with the FSP using a blood drop from an ear vein, a threshold of $51.5\left(\mathrm{Se}=0.71, \mathrm{CI}_{95}=0.62\right.$ to $0.78 ; \mathrm{Sp}=0.66, \mathrm{CI}_{95}=0.53$ to 0.82$)$ and $66.0 \mathrm{mg} /$ $\mathrm{dL}\left(\mathrm{Se}=1.00, \mathrm{CI}_{95}=0.16\right.$ to $1.00 ; \mathrm{Sp}=0.95, \mathrm{CI}_{95}=$ 0.95 to 0.99 ) were determined. The calculated AUC of the thresholds of 51.5 and $66.0 \mathrm{mg} / \mathrm{dL}$ were $0.65\left(\mathrm{CI}_{95}\right.$ $=0.60$ to 0.75$)$ and $0.98\left(\mathrm{CI}_{95}=0.97\right.$ to 1.00$)$, with a $\mathrm{J}$ of 0.36 and 0.95 . Corresponding thresholds for whole blood from the jugular vein were $58.5\left(\mathrm{Se}=0.81, \mathrm{CI}_{95}\right.$ $=0.73$ to $0.87 ; \mathrm{Sp}=0.63, \mathrm{CI}_{95}=0.49$ to 0.79$)$ and $80.0 \mathrm{mg} / \mathrm{dL}\left(\mathrm{Se}=1.00, \mathrm{CI}_{95}=0.16\right.$ to $1.00 ; \mathrm{Sp}=1.00$, $\mathrm{CI}_{95}=0.98$ to 1.00). The AUC of the thresholds of 58.5 and $80.0 \mathrm{mg} / \mathrm{dL}$ were calculated as $0.74\left(\mathrm{CI}_{95}=0.68\right.$ to 0.82$)$ and $1.00\left(\mathrm{CI}_{95}=0.98\right.$ to 1.00$)$, with $\mathrm{J}$ of 0.44 and 1.00 .

\section{Inter- and Intraassay CV}

The average inter- and intraassay CV for determining BHBA concentrations with the FSP were 6.6 and $7.3 \%$, respectively. Corresponding $\mathrm{CV}$ for the GML were 35.6 (interassay) and $35.4 \%$ (intraassay). It should be mentioned that the evaluation of the intra- and interassay agreement had to be repeated, because $80 \%$ of the measurements with the GML device resulted in error messages.

\section{DISCUSSION}

Our study presents a systematic evaluation of the 2 electronic handheld devices FreeStyle Precision and GlucoMen LX Plus for determining BHBA concentrations in goats. The mechanism of subclinical ketosis in goats is not well studied (Andrews, 1997; Sargison, 2007). A therapeutic intervention at an early and moderate stage of the disease, however, is considered as more effective than a treatment of severe HKET (Andrews, 1997; Sargison, 2007). The current study was not designed to demonstrate the prevalence of HKET or the treatment efficacy, but to evaluate the handheld meters for an accurate diagnosis of HKET. The discussion focuses on subclinical HKET with BHBA concentrations $>0.8 \mathrm{mmol} / \mathrm{L}$, because the number of samples with BHBA concentrations $>1.6 \mathrm{mmol} / \mathrm{L}$ was low in our study and none of the does showed any clinical sign of ketosis. Thresholds used in our study were derived from ewes, because no validated BHBA concentrations were available in literature as indicative for HKET in dairy goats. Hence, further studies should focus on evaluating ewe-specific thresholds for HKET, considering their reproductive status. Additionally, further studies may evaluate the accuracy of determined BHBA concentrations from goats suffering from clinical ketosis and the effect of an early intervention on treatment efficacy. 

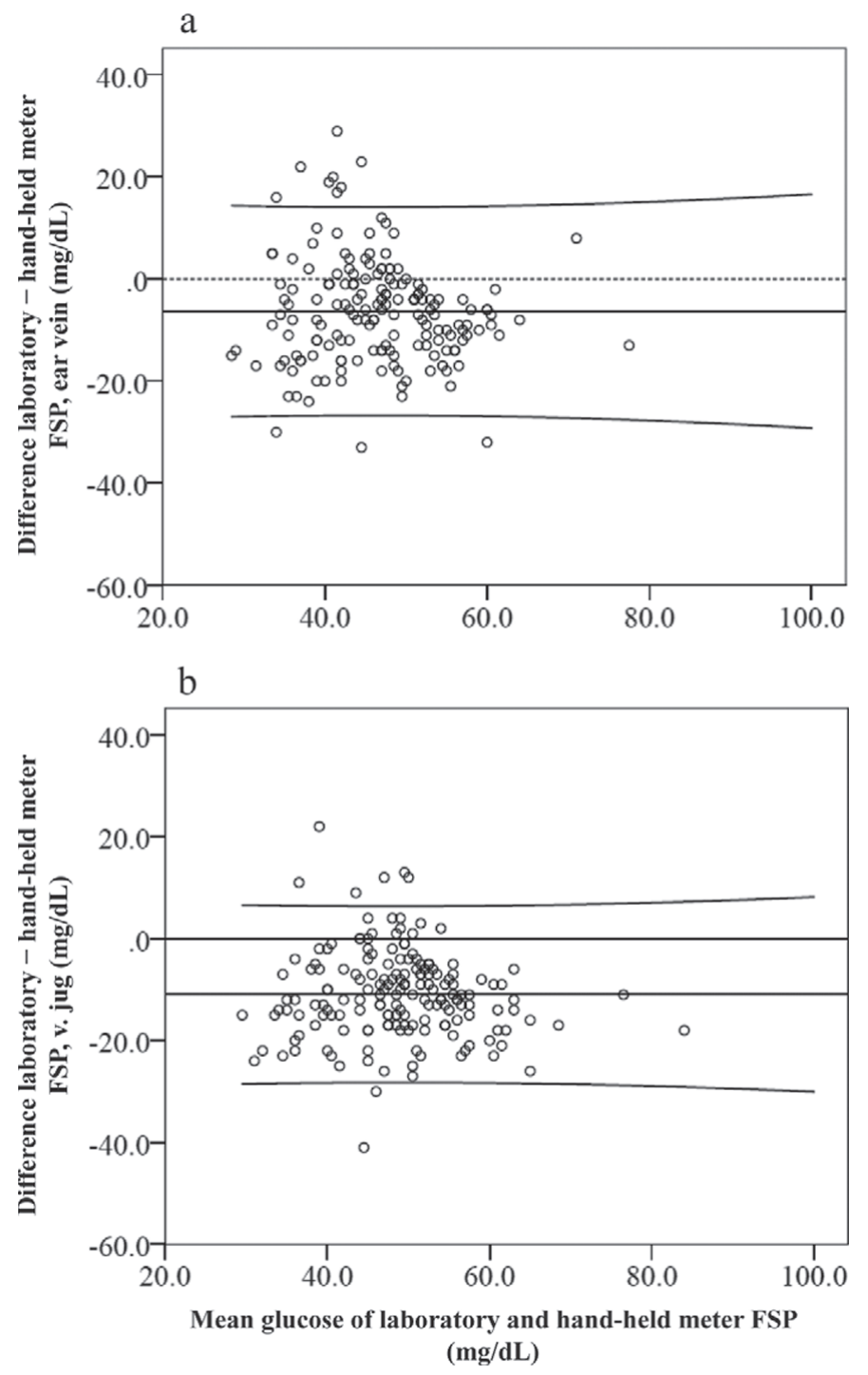

Figure 4. Bland-Altman plot of differences for the FreeStyle Precision handheld device (FSP; Abbott Diabetes Care Inc., Abbott Park, IL) to evaluate the glucose concentration of the whole blood from an ear vein (a) and from the vena jugularis (v. jug.; b). The solid line in the middle shows the mean; the upper and lower solid lines present the mean $\pm \mathrm{SD}$.

As the energy requirements of does considerably increase with the onset of lactation, most does develop a mild or lactational ketosis. Goats in early lactation lose excessive $\mathrm{BW}$ if the feed intake or quality is not adequate to meet the energy requirements. Hence, animals are in a negative energy balance (Matthews, 2009). This could be a major reason for the high percentage $(51 \%, \mathrm{n}=88)$ of samples classified as HKET (BHBA $\geq 0.8 \mathrm{mmol} / \mathrm{L})$ in the present study. Supporting this hypothesis, all of these samples were obtained from animals after kidding.

A strong positive correlation was found for the FSP between the concentration of BHBA analyzed from the whole blood of the jugular vein and the gold standard $\left(\mathrm{r}_{\mathrm{sp}}=0.98\right)$; consistent with the study of Doré et al. $(2013 ; \mathrm{r}=0.98)$. The BHBA determination with the GML presented lower corresponding correlation coefficients of $\mathrm{r}_{\mathrm{sp}}=0.88$. A study on dairy cows by Iwersen et al. (2013) reported similar correlation coefficients for whole blood and serum BHBA concentrations analyzed with the FSP $\left(r_{\mathrm{sp}}=0.94\right)$ and the GML $\left(\mathrm{r}_{\mathrm{sp}}=0.80\right)$ as in the present study.

To our knowledge, ours is the first study in dairy goats that compares the results from 2 different sampling sites (ear vein and jugular vein) for the evaluation of determination of BHBA and glucose concentrations. Using minimally invasive blood sampling techniques, as already established in small animal medicine (Zeugswetter et al., 2010), might also be beneficial in livestock for monitoring of HKET. These sampling sites were chosen because they are commonly used in veterinary practice (jugular vein) or could be easily achieved (ear vein). The correlation coefficient between the BHBA concentration in whole blood of an ear vein and the jugular vein determined with the FSP were high $\left(\mathrm{r}_{\mathrm{sp}}=0.94 ; P\right.$ $<0.01$ ), but lower when measurement was conducted with the GML $\left(\mathrm{r}_{\mathrm{sp}}=0.85 ; P<0.01\right)$.

The correlation between BHBA concentration in serum and in whole blood from an ear vein measured with the FSP was excellent $\left(\mathrm{r}_{\mathrm{sp}}=0.95 ; P<0.01\right)$, indicating that the on-site test is suitable for use in veterinary practice; again, the GML presented a lower corresponding correlation coefficient of $\mathrm{r}_{\mathrm{sp}}=0.85$. The minimal invasive venipuncture of an ear vein was tested in a previous study to identify HKET in ewes and showed similar results for the Precision Xceed $\left(\mathrm{r}_{\mathrm{sp}}\right.$ $=0.94$; Pichler et al., 2014). The venipuncture of goats with small ears might be more challenging and should be tested in further studies.

Bland-Altman plots revealed a positive or negative bias for the FSP and GML compared with the gold standard. The plots of the FSP showed a wider variation with increasing BHBA concentrations. Hence, adjusted clinical decision limits were calculated by ROC analysis. This procedure improved test characteristics for both devices. Test characteristics were slightly better for the FSP than for the GML. This is similar to findings by Iwersen et al. (2013), who also found differences in test characteristics between the 2 devices in dairy cows.

A potential weakness of our study is the number of enrolled animals and the composition of the sample set, which is based on repeated measurements of individual animals. The 11 samples classified as severe HKET, were obtained from 7 animals tested once and from 2 animals tested twice. Calculating the specific Se and Sp for each handheld device by sampling site and time 
revealed an overlapping corresponding $\mathrm{CI}_{95}$ in $95 \%$ (61 of 64) of the compared parameters. Hence, independency was assumed for the corresponding sampling times. However, further studies based on a larger and more diverse sample population should be performed to confirm the evaluated thresholds.

The inter- and intraassay coefficients of variation for the FSP were both below 10\%, which is similar to the recently published data from Doré et al. (2013), who found CV of 5.25 and $3.73 \%$ for a similar device. Coefficients of variation for the GML, however, were both greater than $35 \%$. Reasons for these differences remain speculative and might be related not to the device but to the test strips. The test strip measurement error of the GML during determining the CV was very high $(80 \%)$ and tests had to be repeated. Meanwhile BHBA test strip packages for the GML were modified by the manufacturer, because of their possible effect on the test results. It could be of interest if blood volumes applied to the GML test strips had an effect on the test results. In accordance with the "Guideline on bioanalytical method validation," published by the European Medicines Agency (EMEA, 2011), low, medium, and high BHBA concentrations were chosen for the determination of the CV. Using more samples at different BHBA concentrations with less replications might be beneficial to dilute the effect of any matrix effect. Additionally, further research should be conducted to evaluate the inter- and intraassay CV of the glucose concentration.

In many cases, hyperketonemia is associated with decreased blood glucose concentrations (Andrews, 1997; Rook, 2000; Mavrogianni and Brozos, 2008). These findings are referred to as type I ketosis in dairy cows, when inhibited gluconeogenesis from body protein leads to low plasma glucose levels while the concentrations of ketones are high (Holtenius and Holtenius, 1996). In our study, $27 \%$ of the animals classified as HKET were additionally classified as hypoglycemic.

According to the threshold of $\leq 49 \mathrm{mg} / \mathrm{dL}$ for serum glucose concentrations, $75 \%$ of the serum samples were classified as hypoglycemic. The reason for this finding remains speculative. In serum samples, blood glucose concentrations decrease by ongoing glycolysis, which is dependent on storage temperature (Rehak and Chiang, 1988; Morris et al., 2002). In the present study, clotting was allowed for a maximum of $2 \mathrm{~h}$ and at temperatures between 4 to $10^{\circ} \mathrm{C}$. Hence, under these storage and handling conditions, the effects of glycolysis might be negligible, as reported in the literature (Zhang et al., 1998; Morris et al., 2002). The low concentrations of glucose might also be explained by using serum clot tubes and analyzing serum not plasma. Morris et al. (2002) found higher glucose concentrations in ovine plasma collected with sodium heparinized tubes compared with serum. A study by Kuwa et al. (2001) determined a difference of the glucose concentration between mean values of venous whole blood and venous plasma of $11.3 \%$. Although, in the present study, venous serum was analyzed at the CCPU, it cannot be excluded that collecting systems and substrates have influenced the proportion of samples classified as hypoglycemic.

The correlations between the glucose concentrations determined at the CCPU and in the whole blood of an ear vein or the jugular vein with the FSP were very poor $\left(\mathrm{r}_{\mathrm{sp}}=0.41\right.$ and 0.51 ; both $\left.P<0.01\right)$ and the Bland-Altman plots revealed that the FSP underestimated the glucose concentrations. Even the correlation coefficient for the concentrations determined with the FSP from whole blood of an ear vein and jugular vein was only $\mathrm{r}_{\mathrm{sp}}=0.74(P<0.01)$. Because of the low number of hyperglycemic samples, evaluated thresholds to define hyperglycemia using the FSP are not reliable. Hence, glucose concentrations determined with the FSP should be interpreted with care. Adjusted thresholds for identifying hypoglycemic samples did not result in satisfying test characteristics.

\section{CONCLUSIONS}

After adjusting the thresholds, the FSP device is suitable for analyzing BHBA concentration in goats from the whole blood of the jugular vein and from blood obtained by minimally invasive venipuncture of an ear vein. The GML device showed also good correlations with the gold standard, but the inter- and intraassay coefficients of variation were very high and need further studies for validation. The FSP showed poor test characteristics for determining glucose concentrations and is not suitable for an accurate diagnosis of hypoglycemia.

\section{ACKNOWLEDGMENTS}

We acknowledge the company A. Menarini GmbH (Vienna, Austria) for the financial support and for the test strip supply. Furthermore, we thank the staff of Agriculture Research and Education Center RaumbergGumpenstein for the excellent cooperation.

\section{REFERENCES}

Andrews, A. 1997. Pregnancy toxaemia in the ewe. In Pract. 19:306312.

Bland, J. M., and D. G. Altman. 1986. Statistical methods for assessing agreement between two methods of clinical measurement. Lancet 1:307-310.

Brozos, C., V. S. Mavrogianni, and G. C. Fthenakis. 2011. Treatment and control of peri-parturient metabolic diseases: Pregnancy toxemia, hypocalcemia, hypomagnesemia. Vet. Clin. North Am. Food Anim. Pract. 27:105-113.

Journal of Dairy Science Vol. 97 No. 12, 2014 
Byrne, H. A., K. L. Tieszen, S. Hollis, T. L. Dornan, and J. P. New. 2000. Evaluation of an electrochemical sensor for measuring blood ketones. Diabetes Care 23:500-503.

Carrier, J., S. Stewart, S. Godden, J. Fetrow, and P. Rapnicki. 2004 Evaluation and use of three cowside tests for detection of subclinical ketosis in early postpartum cows. J. Dairy Sci. 87:3725-3735.

Doré, V., J. Dubuc, A. M. Belanger, and S. Buczinski. 2013. Short communication: Evaluation of the accuracy of an electronic onfarm test to quantify blood beta-hydroxybutyrate concentration in dairy goats. J. Dairy Sci. 96:4505-4507.

EMEA (European Medicines Agency). 2011. Guidelines on bioanalytical method validation. European Medicines Agency, London, UK. Accessed June, 25, 2014. http://www.ema.europa.eu/docs/en_GB/ document_library/Scientific_guideline/2011/08/WC500109686. pdf.

Holtenius, P., and K. Holtenius. 1996. New aspects of ketone bodies in energy metabolism of dairy cows: A review. Zentralbl. Veterinarmed. A $43: 579-587$.

Ismail, Z. A., A. M. Al-Majali, F. Amireh, and O. F. Al-Rawashdeh. 2008. Metabolic profiles in goat does in late pregnancy with and without subclinical pregnancy toxemia. Vet. Clin. Pathol. 37:434-437.

Iwersen, M., D. Klein-Jöbstl, M. Pichler, L. Roland, B. Fidlschuster, I. Schwendenwein, and M. Drillich. 2013. Comparison of 2 electronic cowside tests to detect subclinical ketosis in dairy cows and the influence of the temperature and type of blood sample on the test results. J. Dairy Sci. 96:7719-7730.

Krogh, M. A., N. Toft, and C. Enevoldsen. 2011. Latent class evaluation of a milk test, a urine test, and the fat-to-protein percentage ratio in milk to diagnose ketosis in dairy cows. J. Dairy Sci. 94:2360-2367.

Kuwa, K., T. Nakayama, T. Hoshino, and M. Tominaga. 2001. Relationships of glucose concentrations in capillary whole-blood, venous whole-blood and venous plasma. Clin. Chim. Acta 307:187-192.

Matthews, J. 2009. Chronic weight loss. Pages 123-147 in Diseases of the Goat. 3rd ed. J. Matthews, ed. Wiley-Blackwell, Oxford, UK.

Mavrogianni, V. S., and C. Brozos. 2008. Reflections on the causes and the diagnosis of peri-parturient losses of ewes. Small Rumin. Res. 76:77-82.

Morris, J. D., J. M. Fernandez, A. M. Chapa, L. R. Gentry, K. E. Thorn, and T. M. Weick. 2002. Effects of sample handling, processing, storage, and hemolysis on measurements of key energy metabolites in ovine blood. Small Rumin. Res. 43:157-166.

Navarre, C. B., A. N. Baird, and D. G. Pugh. 2012. Diseases of the gastrointestinal system. Pages 71-105 in Sheep and Goat Medi- cine. 2nd ed. D. G. Pugh and A. N. Baird, ed. Elsevier Inc., Maryland Heights, MO.

Noyes, K. J., P. Crofton, L. E. Bath, A. Holmes, L. Stark, C. D. Oxley, and C. J. Kelnar. 2007. Hydroxybutyrate near-patient testing to evaluate a new end-point for intravenous insulin therapy in the treatment of diabetic ketoacidosis in children. Pediatr. Diabetes $8: 150-156$.

Panousis, N., C. Brozos, I. Karagiannis, N. D. Giadinis, S. Lafi, and M. Kritsepi-Konstantinou. 2012. Evaluation of Precision Xceed ${ }^{\circledR}$ meter for on-site monitoring of blood beta-hydroxybutyric acid and glucose concentrations in dairy sheep. Res. Vet. Sci. 93:435-439.

Pichler, M., A. Damberger, I. Schwendenwein, J. Gasteiner, M. Drillich, and M. Iwersen. 2014. Thresholds of whole-blood $\beta$-hydroxybutyrate and glucose concentrations measured with an electronic hand-held device to identify ovine hyperketonemia. J. Dairy Sci. 97:1388-1399.

Rehak, N. N., and B. T. Chiang. 1988. Storage of whole blood: Effect of temperature on the measured concentration of analytes in serum. Clin. Chem. 34:2111-2114.

Rook, J. S. 2000. Pregnancy toxemia of ewes, does, and beef cows. Vet. Clin. North Am. Food Anim. Pract. 16:293-317.

Sargison, N. D. 2007. Pregnancy toxaemia. Pages 359-363 in Diseases of Sheep. 4th ed. I. D. Aitken, ed. Blackwell Publishing Ltd., Oxford, UK.

Schwendenwein, I. 2009. Probenahme, Laboruntersuchungen und invasive diagnostische Maßnahmen. Untersuchung des Blutes. Pages 432-461 in Klinische Propädeutik der Haus- und Heimtiere. 7th ed. W. Baumgartner, ed. Parey, Stuttgart, Germany.

Stelletta, C., M. Gianésella, and M. Morgante. 2008. Metabolic and nutritional diseases. Pages 263-283 in Dairy Goats Feeding and Nutrition. 1st ed. A. Cannas and G. Pulina, ed. CABI International, Wallingford, UK.

Swets, J. A. 1988. Measuring the accuracy of diagnostic systems. Science 240:1285-1293.

Voyvoda, H., and H. Erdogan. 2010. Use of a hand-held meter for detecting subclinical ketosis in dairy cows. Res. Vet. Sci. 89:344351.

Youden, W. J. 1950. Index for rating diagnostic tests. Cancer 3:32-35.

Zeugswetter, F. K., L. Rebuzzi, and S. Karlovits. 2010. Alternative sampling site for blood glucose testing in cats: Giving the ears a rest. J. Feline Med. Surg. 12:710-713.

Zhang, D. J., R. K. Elswick, W. G. Miller, and J. L. Bailey. 1998. Effect of serum-clot contact time on clinical chemistry laboratory results. Clin. Chem. 44:1325-1333. 